\title{
Virtual Technologies in the Medical Professions - Creation of 360 - Degree Environments for Health Care Training
}

\author{
Despina Georgieva, Greta Koleva, Irinka Hristova \\ Ruse University „Angel Kanchev“, Department of Healthcare, Studentska-8 Street, Ruse, Bulgaria
}

\begin{abstract}
This article presents a study of the advantages of virtual technologies in health education, as well as an attempt to answer the following questions. How XR could improve the educational process by boosting the current ratio of students to lecturers while maintaining the same level of efficacy. How XR technologies could provide access to critical knowledge around the clock without interfering with the workflow of healthcare facilities and without exposing patients to any undue risk. The practical application of a $360^{\circ}$ virtual world for a clinical laboratory, central laboratory for sterilization and a virtual operating room all in partnership between the Healthcare Department of Angel Kanchev University of Ruse, The Yatrus Foundation IT Specialists and University Multidisciplinary Hospital for Active Treatment Kanev, Ruse. The virtual reality allows the trainees to explore, to make mistakes and learn from them before ultimately applying the real procedure on a patient.
\end{abstract}

Keywords - nurse, training, augmented reality, virtual reality, 360-degree training environments.

\section{Introduction}

The virtual technologies enter rapidly in our daily routine - in the way of communication, training and the working environment.

DOI: 10.18421/TEM103-39

https://doi.org/10.18421/TEM103-39

Corresponding author: Irinka Hristova,

Ruse University "Angel Kanchev", Department of Healthcare, Studentska-8 Street, Ruse, Bulgaria.

Email: ihristova@uni-ruse.bg

Received: 07 June 2021.

Revised: 01 August 2021.

Accepted: 07 August 2021.

Published: 27 August 2021.

(c) BY-NC-ND (C) 2021 Despina Georgieva, Greta Koleva \& Irinka Hristova; published by UIKTEN. This work is licensed under the Creative Commons AttributionNonCommercial-NoDerivs 4.0 License.

The article is published with Open Access at www.temjournal.com
The EC has approved a Learning Action Plan for introducing both the informational and communication technologies in education. The main goals of this plan are: Developing new standards of training and providing high- quality education system, via creating a virtual European educational space [4]. The Bulgarian education system is based on good traditions, and the experience of the European Community.

The use of virtual worlds in electronic education is quite significant for encouraging the constructive education process. The virtual world provides a new type of interactions and better training practices. The popularity of computer games among children today, and the virtual worlds' educational possibilities, do favour the inclusion of XR technologies in the training process. The specially designed virtual milieus for education comprise spheres in which lots of trainees interact with each other in a virtual 3D world, pursuing a specific educational goal. The training process may be held in a virtual environment, quite similar to the real one, or according to a certain scenario, similar to roleplays [10].

Training in medical specialties is most often carried out as the trainer demonstrates and the student repeats the shown technique or action. This learning process is traditionally conducted in the context of real patient care, which inevitably leads to the risk of increasing medical errors as the learner practices his skills. In the last two decades, simulated environments have been used in medical education as a way to increase the competence of students and at the same time reduce medical errors. This way of learning not only increases the safety of patients, but also improves the clinical skills of the learner, allowing multiple repetitions of the intended skills, combined with specific and informative feedback, which leads to better acquisition of skills. It has been proven that simulation improves teamwork, performing high-risk activities in the training of anaesthesiology, surgery, obstetrics, emergency medicine, paediatrics, resuscitation etc. [12]. 
Although learning through medical simulation is an effective and well-established learning tool, a number of researchers also point out their shortcomings [3], [9].

Virtual reality (VR) based medical education offers the potential to solve a number of problems arising from traditional simulation constraints. VR allows learners to immerse themselves in an authentic and accessible clinical scenario using 360-degree video VR and audio technology (cineVR) without compromising patient safety [6], [13].

Unlike traditional simulation, 360-degree video education provides flexibility, independent learning, and an unlimited number of opportunities to practice and improve teamwork and practical skills applied to patients. The learner interacts with the virtual and authentic real-world environment of 360-degree video, and this allows learners to gain knowledge of the clinical environment before the actual visit. An increasing number of academic medical structures are using VR to enrich their curricula [1], [2], [5], [11].

Unfortunately, the training process of the Health care is one of those sectors in Bulgaria, in which the virtual innovations are still very little applied. The tutors of health care apply different innovative and interactive methods, in order to improve the quality of education and its effectiveness. Video films are used in the training of philosophy and introduction in nurse cares, cases and clinical ones [14], mind maps, and problem-based training. In the training process of palliative care, specially developed protocols of monitoring clinical symptoms are used, standardized scales for assessment of pain, assessment of risk of decubitus, etc. For assessing skills, protocols are being developed and applied.

The project FUTURE EDU of the University of Applied Science in Savonia, Finland, Foundation Yatrus, in partnership with the Chair of Health care in the Ruse University of Angel Kanchev, is aimed at creating virtual environments, which allow training process regardless of place and time, lifelong training for professionals and online training in a realistic environment. Those environments improve both the diversity of training, and the quality of health education. The virtual academic environments use real spheres that cope with professional life, by taking the opportunities of different types of XR technologies. The growing shortage of certified health professionals on the labour market, requires improvement of quality, as well as increasing the access to education. Extended XR and virtual reality (VR) are seldom used in healthcare education, especially in the nurse education. The academic environments that use these new technologies, do expand the traditional methods. They provide safe working environment, and safety for the patients.
This way the participants get a feedback for their representation, and point out exactly what is learned properly, and what needs to be improved. The studies also show that by observing other exercises, one can also learn well, so this is a versatile method of training. The traditional forms and means of training nurses will be diversified with web-based clinical instructions, and virtual-based environments. This will change our teaching strategy and affect the curriculum of nurse education, thus bringing new possibilities of training without interrupting work [8].

\section{Statement}

The goal of the current scientific report is to introduce the advantages of virtual technologies in the training of Health care, and trying to answer a number of questions, concerning the problems of the professional field:

- How will the professions of Nurse and Midwife be more accessible, attractive and safe for the students, by introducing those technologies?

- How will XR facilitate the training process and increase one tutor's capacity to train more students simultaneously?

- How will the XR technologies increase the 24hour access of the information needed, without overloading the working process in hospitals?

The tasks of the current report are the following:

- Explaining the differences between the various types of realities, used in the medical training process.

- Determining the benefits of digitalization of the hospital environments.

- A practical application: introduction of 360degree environments in the partnership of tutors from the University of Applied Science in Savonia, the Ruse University Angel Kanchev, IT specialists from Foundation Yatrus, and the University Hospital Kanev in Ruse.

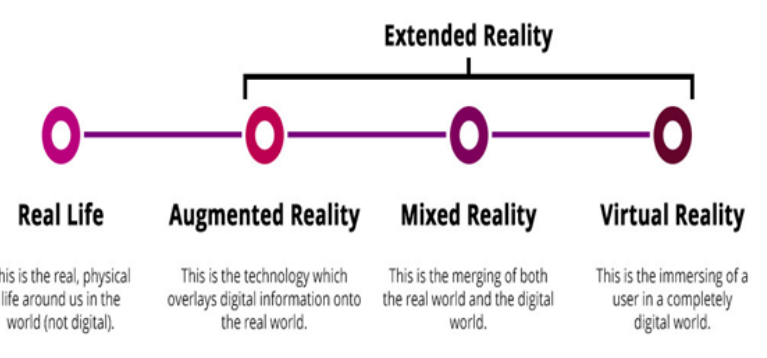

Figure 1. Types of realities

$\mathrm{XR}$ is defined as a main term used to describe the so-called "dipping" technologies, which can combine both the real and the virtual world. They are the well-known today: VR (virtual reality), AR 
(augmented reality) AD (added reality) and MR (mixed reality) plus those that are about to be developed. All those technologies expand the reality which we study.

In the two ends of the line of reality are positioned both the real life RL and virtual reality VR (Figure 1.). $R L$ is everything surrounding us, all that we can see, touch, feel aroma and contact. All our interactions in RL have aftermath. VR is the absolute opposite of it:

- VR is a fully digital environment. The user needs to put on VR headphones and/or mounted display in order to receive a 360-degree view of the virtual world. This way the user may have "the feeling that he's there".

- AR expands the real life with digital details such as photos, text, video, animations, etc. The virtual information and objects lay onto the real world, that is, the users can interact with real life and see what's happening before them. Access can be made via AR goggles or either screens, tablets and smartphones.

- MR is the newest "dipping" technology. In mixed reality, the real world and the virtual one can interact with each other in real time. MR headphones are required for one to experience this. The dip is defined as if it "surrounds the player, the audience etc., so they could feel as like they're fully engaged with something".

- The 360-degree video clips are records in which a view is recorded in a number of directions at one and the same time. 360-degree video clips are often called dipping video clips or spherical video clips. They're usually shot with a specialized undirected camera, or few separately connected cameras, mounted like a spherical massive. 360-degree video clips can be either interactive, or non-interactive. The noninteractive 360-degree videos cannot influence the viewer's experience, whereas the interactive ones can interact with the elements, using either a look or a controller.

VR is an instrument used as a high-technology solution in the field of health care, for improving medical education. The continuing post-graduate training in the field of health care is of high importance for the medical specialists. The health industry develops constantly, new techniques and technologies appear, which necessitates the medical specialists to regularly follow those innovations, so they could expand both their skills and knowledge.

Application of VR technologies in developing scenarios for simulation of trauma and emergency. Working in emergency wards requires skills, quick thinking and the ability to function under high stress. At the emergency ward in Stanford in a new simulation of training, a VR scenario is developed which puts the doctor in a situation where a patient needs immediate medical aid, but the doctor is being interrupted 12 times during the patients' check-up. The goal is to recreate this in real life, where the physicians are faced with this scenario, and such interruptions may increase the risk of errors. Using the VR, they can practice and be trained to solve a lot of cases in a busy, high-risk and stress environment.

$A R$ as an aid during work/practice/training. $A R$ applications provide information in real time that is focused on physical objects, which simplifies the process of perceiving the information. How exactly will the expanded reality find its way in practical exercises in the anatomy of the digestive system, where the lectures are usually given with static or printed images. Any student can direct a screen toward a physical object or even AR marker, so he can see the content in graphic animation. By this method, the learning process raises up to $40 \%$, and the logical connections between a subject and some information about it in the brain become much more durable [7].

Developing 360-degree environments for training purposes - shooting of hospital environments. One of the main problems in entering a real hospital environment is that the students' groups need not consist of more than 6-8 students who have to be present meanwhile in an operating room, clinical lab, sterilization lab, etc., without that foiling the quality of performing both the working and the training process. This, on the other hand, leads to organization of multiple visits, repetition of one and the same information for each separate group, as well as high expenses on providing safety on both the students, and the environment. The hospital is a place of high intensity of work, and every day is different than yesterday, which leads to extreme situations following the training process, to its impossibility for holding it.

A team of agents from the University of Applied Science in Savonia, Finland, Foundation Yatrus, and tutors from the Chair of Health care and from the University Hospital Kanev, are putting the finishing touches on three different 360-degree environments, for training purposes.

\section{0-degree environment for training in Matterport and Thinglink:}

- A safe walk in a clinical lab;

- A central sterilization lab;

- A virtual operating room.

Materials and methods: Technical support for developing 360-degree environments for training purposes: 
- A 360-degree camera - there's a big variety of cameras in the global market. Some of the best ones for 2020 are: Insta 360, RICHOH THETA Z1 and Matterport pro 2. The environments created in the University Hospital Kanev in Ruse are shot with RICHOH THETA V, the best 360 camera for 2019.

- A smartphone cell phone with a 360-degree camera management software, aiming to avoid the presence of people in the footage. The cameras are equipped with a wi-fi connection to which the phone is linked. Every producer provides a software for camera management that allows setting corrections such as: HDR, ISO, $\mathrm{EV}$, in order to attain the best quality of the picture.

- A stand - it's important for it to have indexes that show its levelling. The goal is for the photo to have a perfectly right horizon, it's of great importance if the environment is to be visualized by VR goggles. The lack of photo levelling would cause vertigo and no feeling of balance for the one wearing those VR goggles.

\section{Photo-processing software:}

Photoshop: its latest versions allow easy dealing with 360-degree photos. Either a text or images may be added without the photos being curved subsequently in visualizing the complete 360 -degree environment. Photoshop also provides the possibility of removing unwanted elements from the flash such as the stand, passers-by (if the photos are taken outdoors).

Thinglink - an online platform designed for creating an interactive 360-degree environment. The photos are first uploaded as a 360 image/video, and then linked to interactive points that allow transition from frame to frame, aiming to represent a virtual stroll. The platform also gives the possibility of adding interactive points containing: a text, photos, drawings, video, link (leading to additional information), as well as integrating a closed-answer test. The environments are accessible via smartphone cell phone, computer, laptop, tablet, and VR goggles. The device needs to have Internet access, and you to have an access link. The environments are best operated via computer and tablet, but it's absolutely no problem for one to interact with them through the phone, too. For visualization through VR goggles, a little more experience is necessary to deal with the environments' navigation.

360-degree environment for training in Matterport and Thinglink: The central sterilization room is assembled on 8 individual 360-degree photos, it's got more than 15 text informational points, denoted with blue book and 6 videos showing the sterilization process (Figure 2.).
The operating room (Figure 4.) and the Clinical lab (Figure 3.) are shot at the University Hospital Kanev, while the information for each of the informational points is being provided by the Chair of Health care.

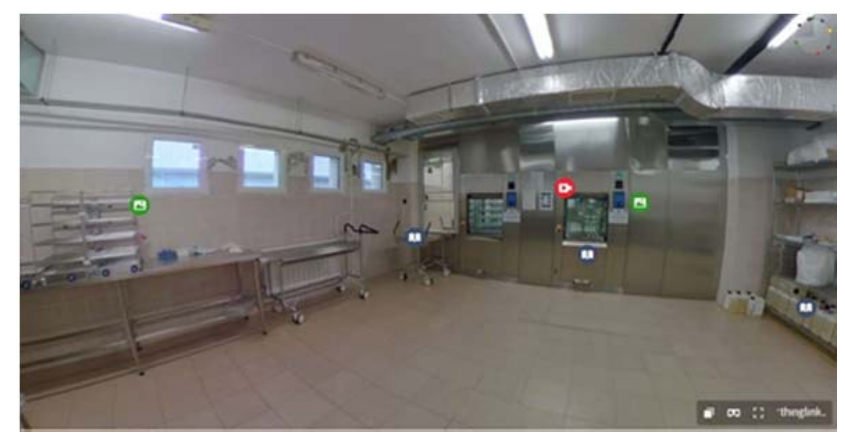

Figure 2. 360- degree environment for training in Matterport and Thinglink: Central sterilization



Figure 3. 360- degree environment for training in Matterport and Thinglink: Clinical lab

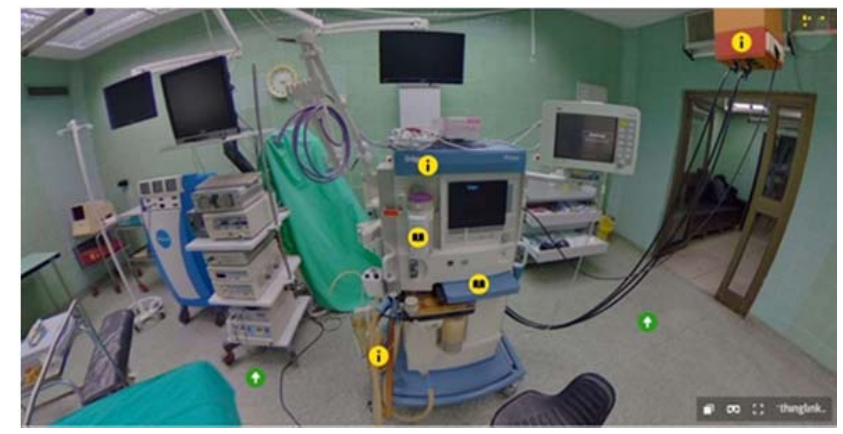

Figure 4. 360-degree environment for training in Matterport and Thinglink: Operating room

\section{Conclusion}

The effective mastering of knowledge will allow the nurses learning to perform interdisciplinary links concerning the scientific theoretical facts, to analyse and synthesize information, to make correct assessments, to develop clinical way of thinking, to apply theoretical knowledge in practical training, to suggest solutions to specific problems, and to link what they have already learned with new knowledge. The training is bound with clinical practice from the very first semester on, this requires active, constant and effective learning, and exercise of practical skills. 
The advantages of the 360-degree shooting of hospital environments, does not aim complete substitution of practical training in a real environment, just its optimization. The other important advantage is the opportunity for the students to get general information of the hospital environment, which allows faster implementation of the practical exercises that require multiple reiterations, as well as tutor's explanations.

The use of the VR for medical training has great potential in the teaching process of the nurses, aimed at learning a number of procedures and manipulations, which are to be performed perfectly, safely and quickly enough. By using the XR technologies, the students can perform those procedures in a completely captivating simulation, which produces scenarios from the real world. XR technologies do assist the traditional training process, thus giving the opportunity for doing as much repetitions as needed for learning a technique or algorithm of medical activity, before applying it to a patient. The students are traditionally trained by dummies, by specially designed training software, clinical cases in real environment, as well as other resources of practical training. All of these resources have their proven unvarying place, but can be successfully expanded by the VR. The virtual reality allows the trainees to explore, to make mistakes and learn from them before ultimately applying the real procedure on a patient.

The created 360-degree environments are used in the education of students majoring in Nursing. The results of the conducted research for:

- the effective acquisition of knowledge, formation of skills and competencies in the field of organization and work of the sterilization department, operating room and clinical laboratory;

- degree of perception of the provided information;

- students' satisfaction with the training with 360degree environments;

- opportunities for learning not only in the regulated study time.

Will be published in the next scientific publication.

\section{References}

[1]. Baker, M. (2017). How VR is revolutionizing the way future doctors are learning about our bodies. University of California San Francisco, 18.

[2]. Buchman, S., Miller, C., Henderson, D., Williams, E. R., \& Ray, S. (2020). Interprofessional Students Learning to Save a Life through Cine-VR Simulation. EC Nursing and Healthcare,22(11), 2020 .
[3]. Carron, P. N., Trueb, L., \& Yersin, B. (2011). Highfidelity simulation in the nonmedical domain: practices and potential transferable competencies for the medical field. Advances in medical education and practice, 2, 149.

[4]. Commission of the European Communities. (2001). The E-learning Action Plan: Designing Tomorrow's Education. Commission of the European Communities.

[5]. Erickson, M. (2017). Virtual reality system helps surgeons, reassures patients. Stanford Medicine.

[6]. Harrington, C. M., Kavanagh, D. O., Ballester, G. W., Ballester, A. W., Dicker, P., Traynor, O., ... \& Tierney, S. (2018). 360 operative videos: a randomised cross-over study evaluating attentiveness and information retention. Journal of surgical education, 75(4), 993-1000.

[7]. Herault, R. C., Lincke, A., Milrad, M., Forsgärde, E. S., \& Elmqvist, C. (2018). Using 360-degrees interactive videos in patient trauma treatment education: design, development and evaluation aspects. Smart Learning Environments, 5(1), 1-15.

[8]. Mähönen, A., Halimaa, S., Kontro, P., \& Pakarinen, K. (2020). Work life related virtual learning (XR) environments for biomedical laboratory science and perioperative nursing in the futureedu project. Journal of International Scientific Publications: Educational Alternatives, 18, 105-115.

[9]. Okuda, Y., Bryson, E. O., DeMaria Jr, S., Jacobson, L., Quinones, J., Shen, B., \& Levine, A. I. (2009). The utility of simulation in medical education: what is the evidence?.Mount Sinai Journal of Medicine: A Journal of Translational and Personalized Medicine: A Journal of Translational and Personalized Medicine, 76(4), 330-343.

[10]. Pagani, M. (Ed.). (2008). Encyclopedia of multimedia technology and networking (Vol. 3). IGI Global.

[11]. Palter, V. N., \& Grantcharov, T. P. (2014). Individualized deliberate practice on a virtual reality simulator improves technical performance of surgical novices in the operating room: a randomized controlled trial. Annals of surgery, 259(3), 443-448.

[12]. Patel, D., Hawkins, J., Chehab, L. Z., Martin-Tuite, P., Feler, J., Tan, A., ... \& Sammann, A. (2020). Developing Virtual Reality Trauma Training Experiences Using 360-Degree Video: Tutorial. Journal of medical Internet research, 22(12), e22420.

[13]. Pulijala, Y., Ma, M., Pears, M., Peebles, D., \& Ayoub, A. (2018). Effectiveness of immersive virtual reality in surgical training - a randomized control trial. Journal of Oral and Maxillofacial Surgery, 76(5), 1065-1072.

[14]. Hristova, T. (2019). Application of case study method in crew members' training program. Scientific Bulletin" Mircea cel Batran" Naval Academy, 22(1), 0_1-7. 